\title{
Génération de vibrations par les couches limites turbulentes
}

\author{
D. Leducq Société Bertin et Cie \\ R. Perret Société Alsthom - ACB - CERG
}

L'écoulement turbulent au voisinage d'une paroi est le siège de fluctuations dynamique qui se traduisent sur la paroi par un champ de forces aléatoires convecté à une vitesse voisine de celle de l'écoulement. La structure spatiale et temporelle de cette excitation est directement liée à celle de la couche limite et dépend de son organisation. On passe en revue les différents modèles dérivés de l'analyse expérimentale des couches limites turbulentes. La production de vibrations dépend de la capacité de la structure à transformer l'énergie cinétique d'excitation en énergie vibratoire; cette capacité est plus ou moins forte suivant la coïncidence en nombre d'ondes et fréquences de l'excitation et des modes propres à la structure. Au vu de ces considérations, on dégage les axes à suivre pour réduire le niveau de vibrations induit par une couche limite turbulente.

1. Génération des fluctuations de pression hydrodynamique

\subsection{Introduction}

En dehors des obstacles tels que vannes, changements de section, coudes, embranchements ou des organes actifs, l'écoulement en conduite hydraulique est un écoulement turbulent développé que l'on peut caractériser vis-à-vis de son interaction avec la paroi par la présence d'une couche limite où l'écoulement passe d'une vitesse nulle à la paroi, à la vitesse " axiale " maximale.

Cette couche limite est le siège de mouvements hydrodynamiques fluctuants qui créent des fluctuations de pressions au-dessus de la paroi où ils se produisent; ces fluctuations, intégrées sur la surface où elles sont cohérentes, vont produire un champ de forces aléatoires convecté avec l'écoulement qui va exciter les vibrations de la structure. C'est cette excitation hydrodynamique que nous allons décrire dans cette première partie.

\subsection{L'équation du champ de pression}

A partir des équations de conservation de la masse et de la quantité de mouvement, associées à une équation d'état, on obtient l'équation classique de Lighthill :

$$
\nabla_{p}^{2}-\frac{1}{c^{2}} \cdot \frac{\partial^{2} p}{\partial t^{2}}=-\frac{\partial^{2} \rho u_{i} u_{j}}{\partial x_{i} \partial x_{j}}
$$

où $p$ est la pression instantanée, $\rho$ la den sité $c$ la célérité du son, $u_{i}$ la composante de la vitesse instantanée dans la direction

\section{Generation of vibrations by turbulent boundary layer.}

The turbulent flow close to a wall is the source of dynamic fluctuations expressed on the wall by a random forces' field convected at a speed approaching that of the flow. The spatial and time structure of the excitation is related to that of the boundary layer and depends on its organization. Various models derived from the experimental analysis of turbulent boundary beds are reviewed. The generation of vibrations depends on the capacity of the structure for transforming the excitation kinetic energy into vibratory energy. This capacity is more or less strong, depending on the coincidence of the wave numbers and excitation frequencies with the modes belonging to the structure. In the light of these considerations, guidelines to be followed are suggested for reducing the level of vibrations induced by a turbulent boundary layer. 


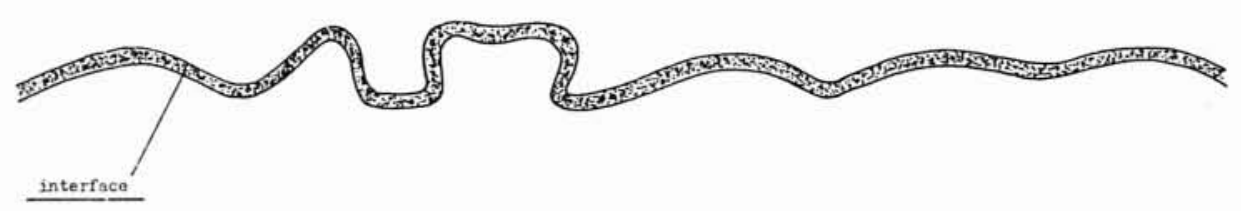

2ONE DE SILLAGE

$0<r<1$

1.

OR 1 <1

2.

$\overline{\mathrm{h}}=0,7 \delta$
$\overline{\mathrm{I}}=2 \delta$
$\overline{\mathrm{L}}=48$ = dimenaion tranaversale

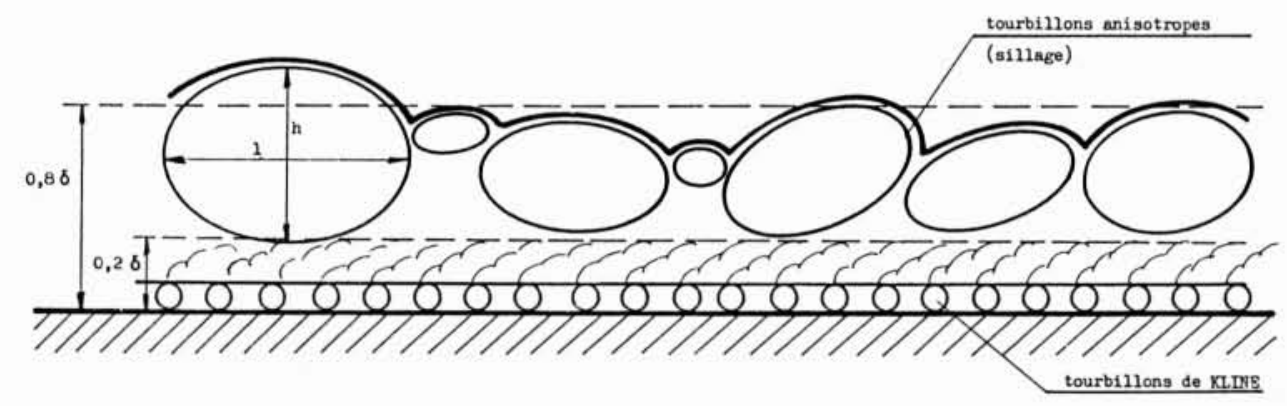

3.

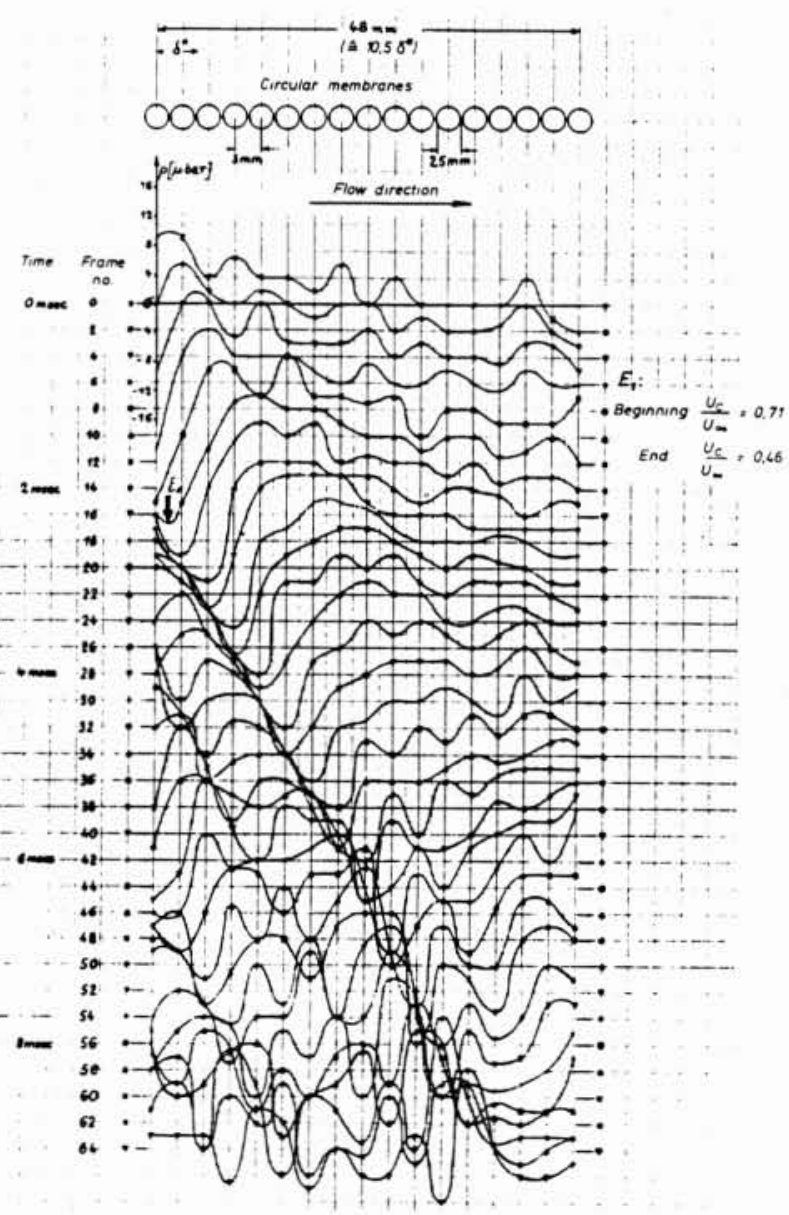

1. Structure de la couche limite turbulente.

2. Modèle physique des sources de pression dans une couche limite turbulente.

3. Evolution spatio-temporelle de la pression sur une génératrice de cylindre.

4. Mesure du champ de pression pariétal 2-D (Emmerling 1973).

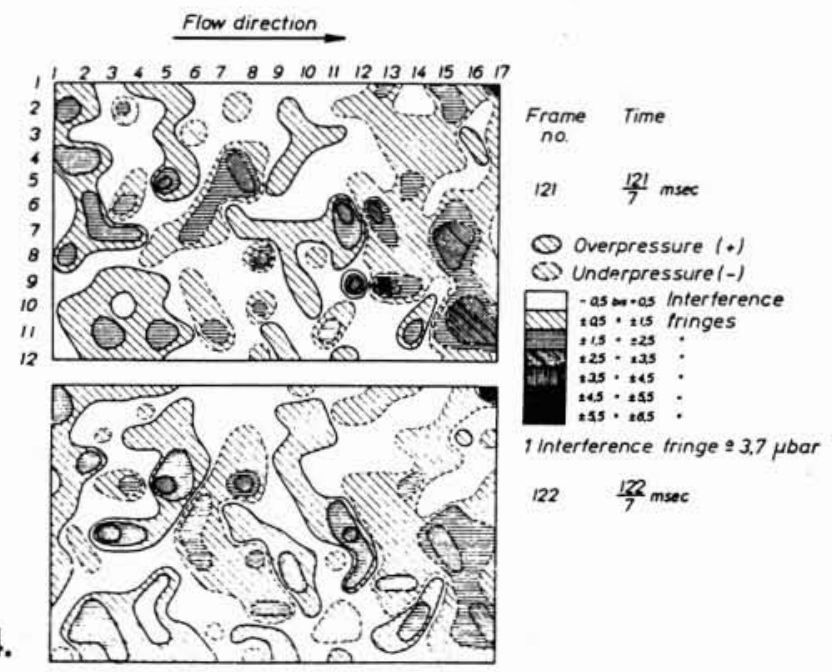


Dans les écoulements hydrauliques couramment rencontrés, les vitesses d'écoulements sont faibles par rapport à $c(c \simeq 1500 \mathrm{~m} / \mathrm{s})$, et l'effet de compressibilité peut être négligé dans la formulation du champ de pression excitateur de la paroi en vibration. (1) se réduit alors à :

$$
\nabla_{p}^{2}=-\frac{\partial^{2} \rho u_{i} u_{j}}{\partial x_{i} \partial x_{j}}
$$

Dans la couche limite, avec une vitesse moyenne d'écoulement $U, u_{1}=U+u^{\prime}$ et une vitesse normale à la paroi $u_{z}=v^{\prime}$, l'équation (2) se simplifie en:

$\frac{1}{\rho} \nabla_{p}^{2}=-\frac{2 \partial U \partial v^{\prime}}{\partial y \partial x}-\frac{\partial^{2}}{\partial x \partial y}\left[u^{\prime} v^{\prime}-\overline{u^{\prime} v^{\prime}}\right]$

$\left(x_{1}=x, x_{z}=y\right)$ réf. 1 .

C'est l'équation (3) qui, intégrée pour $y=0$, donne le champ de pression en parois.

On remarquera que $\partial U / \partial y$ est lié au cisaillement à la paroi par la relation $\tau=\mu(d v / d y)$.

$\rho u^{\prime} v^{\prime}$ est l'expression des tensions de Reynolds. (3) s'écrit alors

$\operatorname{div}(\overrightarrow{\operatorname{grad} p})=-\frac{2 \tau}{\mu} \quad \frac{\partial v^{\prime}}{\partial x}-\frac{\partial^{2}}{\partial x \partial y}\left(\rho u^{\prime} v^{\prime}-\overline{\rho u^{\prime} v^{\prime}}\right)$

(2)

Les termes (1) et (2) sont les termes sources du champ excitateur. Le terme (1) représente l'interaction du cisaillement pariétal avec les fluctuations de vitesses verticales. Le terme (2) les fluctuations spatiales des tensions de Reynolds instantanées. L'intégration de (3) nécessite donc la connaissance de la structure de la couche limite et notamment de son degré d'organisation, qui fera que l'intégrale spatiale de $\partial v^{\prime} / \partial x$ et de (2) sera différente de 0 .

\subsection{La structure de la couche limite [réf. 2]}

Celle-ci n'est pas homogène et on rencontre entre la paroi et sa limite supérieure plusieurs zones présentant un certain degré d'organisation, caractérisé par l'intermittence, rapport du temps où l'écoulement est turbulent au temps où l'écoulement est laminaire.

Les figures 1 et 2 présentent une vue schématique de cette structure. Sur le plan source d'excitation hydrodynamique, 2 zones sont importantes:

a) la sous couche visqueuse, de très faible épaisseur, parcourue par des "tourbillons de Kline" structures très régulières convectées à la vitesse locale (taille de l'ordre de $5 \delta / 100$, vitesse $5 U_{\infty} / 100$ );

b) la couche de sillage, parcourue par des structures dont la dimension caractéristique est de l'ordre de l'épaisseur de couche limite $\delta$ et qui sont convectées à une vitesse voisine de celle de l'écoulement moyen $\left(U_{c} \simeq\right.$ 0,6 à $\left.0,8 U_{\infty}\right)$.

Chaque structure relativement organisée se présente comme une "zone de haute pression" ou de "basse pression ", pour prendre une image météorologique, d'étendue finie dans le sens de l'écoulement et normale-
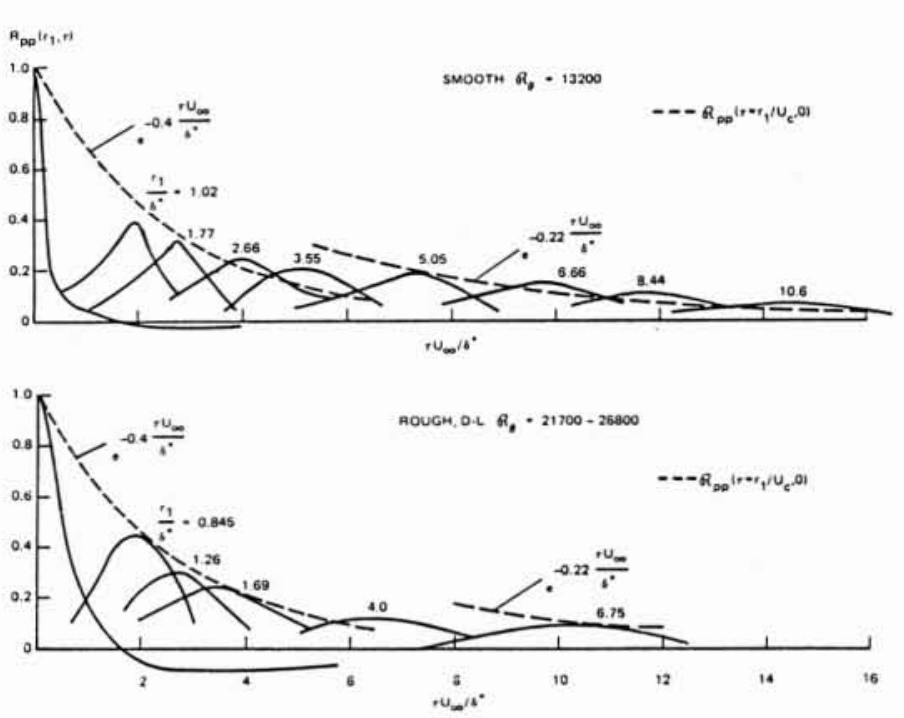

5. Corrélations spatio-temporelles de pression sur parois lisses et parois rugueuses.

ment à celui-ci sur la paroi. Ce sont ces "hautes pressions " et ces " basses pressions " qui, intégrées sur les sections où elles présentent une organisation homogène, génèrent l'excitation de la structure.

La figure 3, tirée d'une étude de Emmerling [réf. 3] montre l'évolution au cours du temps, sur une génératrice de paroi cylindrique, de ce champ de pression. On distingue très bien l'effet de convection et d'évolution au cours du temps.

\subsection{Caractérisation du champ pariétal de pres- sion}

L'expérience d'Emmerling citée ci-dessus consistait, par une méthode optique, à mesurer tout le champ de pression instantanée et son évolution au cours du temps. La figure 4 donne un exemple du résultat obtenu.

Le problème posé ici est en fait de caractériser l'excitation sous une forme compatible avec les techniques d'étude de la réponse vibratoire de la structure: réponse locale, analyse modale, éléments finis...

Pour obtenir la caractérisation spatiotemporelle nécessaire au champ de pression, on passe alors par les mesures de corrélations spatiotemporelles [réf. 5]

$R(\vec{r}, \tau)=\frac{\overline{p(\vec{x}, t) p(\vec{x}+\vec{r}, t+\tau)}}{\overline{p(\vec{x}, t)^{2}}}$

La figure 5 présente un exemple de résultats obtenus pour une paroi lisse et pour une paroi rugueuse.

Chaque courbe présente un maximum pour un décalage temporel $\tau_{m}$. On montre aisément que si les capteurs sont séparés de $\vec{r}$, la vitesse de convection au cours du temps est $\vec{U}_{0}=\vec{r} / \tau_{m}$.

L'enveloppe des courbes de corrélation traduit l'évolution lagrangienne des perturbations au cours du temps.

Les modélisations de ces corrélations ou de leurs transformées de Fourier sont à la base des modèles de sources d'excitation que nous allons passer en revue. 


\subsection{Modélisation du champ de pression}

Elle consiste à quantifier le champ des forces aléatoires convectées avec l'écoulement pour obtenir :

- soit le niveau global en 1 point,

- soit le spectre fréquentiel en 1 point,

- soit la structure spatiotemporelle ou spatiofréquentielle sur 1 domaine donné.

\subsubsection{Modèle de Kraichnan}

Le modèle de Kraichnan donne le niveau global en 1 point.

Kraichnan admet que le terme d'interaction turbulence - turbulence $\frac{\partial^{2}}{\partial x \partial y} \quad\left(u^{\prime} v^{\prime}-\overline{u v}\right)$ de l'équation (4) est négligeable devant le terme d'interaction turbulence cisaillement moyen, $\frac{\partial \bar{U}}{\partial y} \frac{\partial v^{\prime}}{\partial x}$, l'équation (4) s'écrit alors :

$$
\nabla_{p^{\prime}}^{2}=-2 \rho \frac{\partial U}{\partial y} \frac{\partial v^{\prime}}{\partial x}
$$

En partant de cette équation, Kraichnan aboutit à une expression de l'écart type de la pression à la paroi en fonction de la tension de cisaillement à la paroi $\tau_{0}$

$$
\sqrt{\bar{p}^{\prime 2}}=\alpha \tau_{0}
$$

Il faut cependant signaler que d'après Corcos l'approximation réalisée n'est pas très bonne et que dans certains cas, la contribution du terme d'interaction turbulence - turbulence peut atteindre 35 à $40 \%$ de la fluctuation totale de pression.

De nombreuses valeurs divergentes ont été proposées pour Kraichnan donne une valeur comprise entre 2 et 12. Corcos à la suite d'une analyse de différents résultats suggère $3 \pm 0,5$.

Willmarth et Wooldridge [réf. 4] ont observé que $\alpha$ augmentait avec la rugosité.

\subsubsection{Représentation spectrale en un point}

L'analyse dimensionnelle montre que les résultats peuvent se mettre de façon synthétique sous la forme:

$$
\frac{P(\omega) U_{\infty}}{\left(\frac{1}{2} \rho U_{\infty}^{2}\right)^{2} \delta^{\star}}=f\left(\frac{\omega \delta^{\star}}{U_{\infty}}\right)
$$

où $P(\omega)$ est la densité spectrale de puissance de la pression

$\delta^{\star} \quad$ l'épaisseur de déplacement de la couche limite

$\omega=2 \pi f$ la fréquence circulaire.

La figure 6 présente une synthèse des résultats effectués par Bull [réf. 5].

On voit que pour un projet pratique ce spectre peut se schématiser par la représentation suivante :

a) jusqu'à une fréquence de l'ordre de $U / \delta \approx U / \omega \delta^{\star} \# 1$

$$
P(\omega)=P^{2}{ }_{0}=\text { Cte }
$$

b) au-delà, le spectre décroît en $1 / f^{m}, m$ \# 3 (l'exposant $m$ dépend de l'état de surface).
En faisant intervenir le coefficient de frottement $C_{f}$ et le coefficient $\alpha$ de la formule de Kraichnan, on obtient:

$$
\begin{aligned}
& \overline{P(\omega)}=\frac{P(\omega) U}{\left(\frac{1}{2} \rho U\right)^{2} \delta^{*}}=\frac{n-1}{n} \alpha^{2} C f \frac{1}{\omega_{0}} \quad \bar{\omega} \leqslant \bar{\omega}_{0} \\
& \overline{P(\omega)}=\frac{P(\omega) U}{\left(\frac{1}{2} \rho U^{2}\right)^{2} \delta^{*}}=
\end{aligned}
$$

$$
\frac{n-1}{n} \alpha^{2} C f\left(\frac{\overline{\omega_{0}}}{\omega}\right)^{n} \frac{1}{\omega_{0}}
$$

pour $\bar{\omega} \geqslant \bar{\omega}_{0}$

\subsubsection{Modèles spatiofréquentiels}

\subsubsection{Modèle de Corcos [réf. 6]}

C'est le modèle le plus utilisé en pratique quand on veut étudier la réponse vibratoire d'une structure excitée par une couche limite turbulente.

Corcos considère la transformée de Fourier fréquentielle de l'intercorrélation $\Phi_{p p}\left(r_{1}, r_{3}, \omega\right)$ et la met sous la forme

$\Phi_{p p}\left(r_{1}, r_{3}, \omega\right)$

$=\Phi_{p p}(\omega) \exp \left(i \omega r_{1} / U_{c}\right) \mathrm{A}\left(\omega r_{1} / U_{c}\right) B\left(\omega r_{3} / U_{c}\right)$

$A$ et $B$ traduisent la cohérence spatiale des sources de pressions. La confrontation avec les résultats expérimentaux donne :

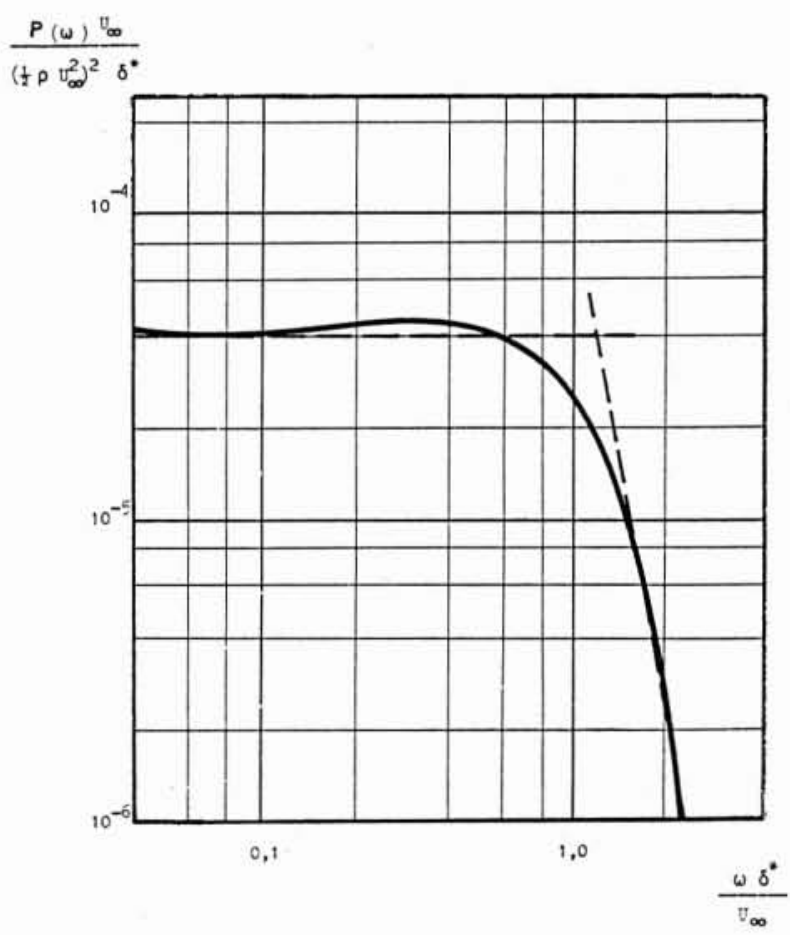

6. Spectre de puissance de la pression à la paroi. 


$$
\begin{aligned}
& A=e^{-\left(\gamma_{1} \eta_{\omega} / v_{c}\right)} \\
& B=e^{\left.-\gamma_{3} r_{3} \omega / v_{c}\right)}
\end{aligned}
$$

$\gamma_{1}$ vaut entre 0,1 et 0,4 : la valeur dépend de l'état de surface

$\gamma_{3}$ vaut environ 0,7 .

1.5.3.2. Passage dans le domaine fréquence Nombre d'ondes

Le vecteur nombre d'ondes $\left(k_{1}, k_{3}\right)$ s'obtient par transformation de Fourier à partir du vecteur d'espace $\left(r_{1}, r_{3}\right)$; la relation (21) se transpose alors sous la forme:

$\Phi_{p p}(\vec{k}, \omega)=\Phi_{p p}(\omega) \varphi_{1}\left(k_{1}\right) \varphi_{3}\left(k_{3}\right) \phi_{m}\left(k_{1}-\frac{\omega}{U_{0}}\right)$

qui s'écrit, avec les hypothèses de Corcos

$$
\begin{aligned}
& \Phi_{p p}(\vec{k}, \omega)=\frac{\left[\Phi_{p p}(\omega)\right]}{\pi^{2}} \gamma_{1} \gamma_{3}\left(\frac{\omega \delta^{*}}{U_{c}}\right)^{2} \delta^{* 2} \\
&\left\{\left[\left(\frac{\gamma_{3} \omega \delta^{*}}{U_{c}}\right)^{2}+\left(k_{3} \delta^{\star}\right)^{2}\right]\right. {\left[\left(\frac{\gamma_{1} \omega \delta^{\star}}{U_{c}}\right)^{2}\right.} \\
&\left.\left.+\left(k_{1} \delta^{*}-\frac{\omega \delta^{*}}{U_{c}}\right)^{2}\right]\right\}^{-1}
\end{aligned}
$$

C'est ce type de fonctions d'excitation que l'on rentrera lorsque l'on voudra étudier la réponse modale de la structure en vibration.

\subsubsection{Modèles de type "éléments finis ou différen- ces finies "}

La réponse de la structure peut également se modéliser par un code d'éléments finis. Il faut dans ces conditions pouvoir modéliser la fonction d'excitation sous la même forme : définir des éléments finis fluides, convectés avec l'écoulement, caractérisés par leurs tailles, l'intensité des pressions convectées, la durée durant laquelle celles-ci sont cohérentes, les lois de pertes de cohérences...

Plusieurs modèles existent ou sont en cours de développement.

Le modèle de U. Schumann, basé sur la résolution de Navier-Stockes en bi-dimensionnel avec une modélisation de la turbulence de type SGS ("Sub. Grid. Scale " : on suppose la turbulence définie par ses caractéristiques moyennes au-delà d'une certaine échelle, de l'ordre de l'échelle intégrale de turbulence) a été proposé en 1975 pour des applications dans le nucléaire [réf. 7].

On modélise ainsi le champ de pression en paroi à chaque instant (fig. 7). Pour y parvenir, Schumann modélise cette fois les fonctions d'inter-corrélations décrivant l'évolution spatio-temporelle des phénomènes.

$\mathrm{Si}$ on rapproche ces prédictions des mesures faites par Emmerling en 1973 (fig. 4), on constate une très bonne concordance, ce qui valide ce genre de modèle.

D'autres modèles décrivent le champ de pression sous forme d'une distribution aléatoire de tourbillons discrets, suivant certaines lois statistiques dérivées également de l'observation des corrélations spatiotemporelles. Un modèle basé sur ce principe avait été proposé en 1974 par la société Bertin.

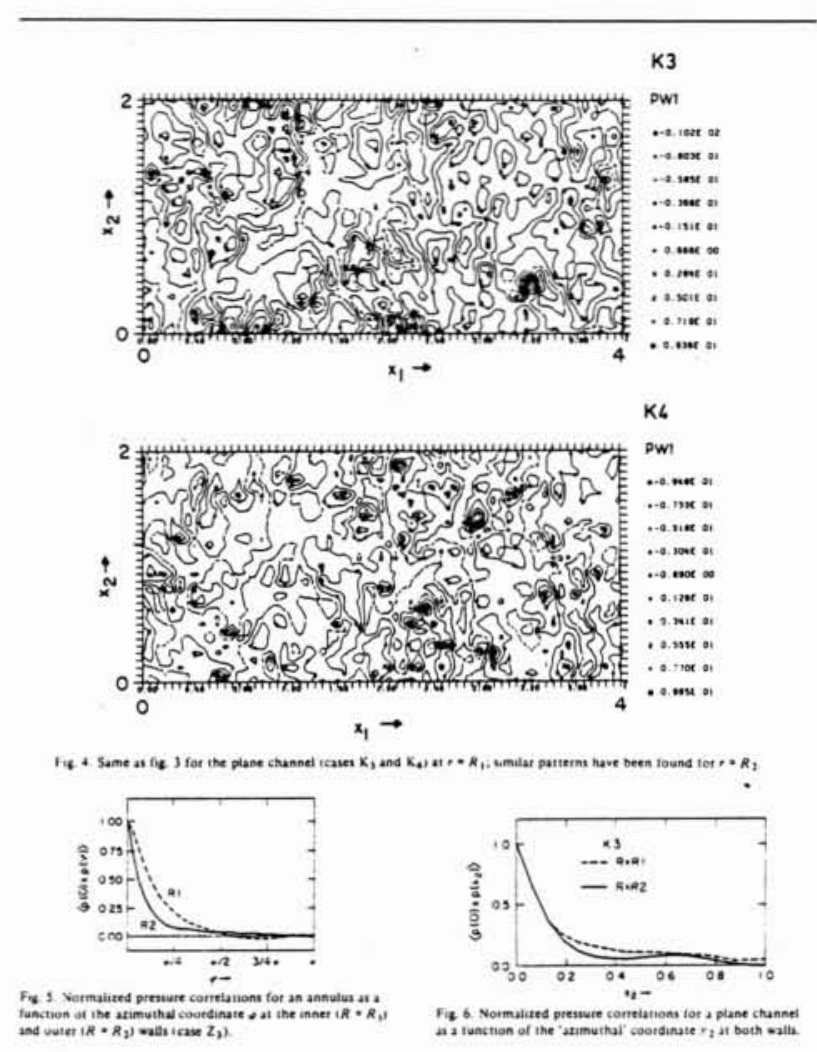

7. Modèle numérique du champ de pression (d'après $U$. Schumman).

\section{Les mécanismes de génération de vibra- tion par excitation de couche limite tur- bulence}

\subsection{Introduction}

La prédiction des phénomènes vibratoires induits sur des structures par des écoulements turbulents suppose d'une part la résolution d'un problème dynamique d'interaction fluide-structure et, d'autre part, la connaissance du champ excitateur. Après la présentation du phénomène d'excitation par la couche limite qui vient d'être faite, il est nécessaire de développer la connaissance de la réponse vibratoire d'une structure soumise à une excitation. De nombreux auteurs ont examiné ce problème. On citera deux contributions récentes et particulièrement importantes, les ouvrages de F. Fahy [8] et de W.K. Blake [9]. Nous présentons ici, appliqués au cas de structures de type plaques planes, les principaux phénomènes apparaissant dans l'interaction fluide-structure, analysés à partir d'une formulation modale du problème qui permet une approche physique.

\subsection{Couplage fluide structure}

Si l'on considère une structure immergée soumise à des efforts ponctuels ou répartis, il se produit un couplage entre le fluide et la structure que l'on peut définir comme la modification du champ de force excitateur dû, soit aux déformations de la structure, soit au champ de pression dans le fluide induit par les déformations de la structure. 
Le schéma ci-après précise cette définition. (1)

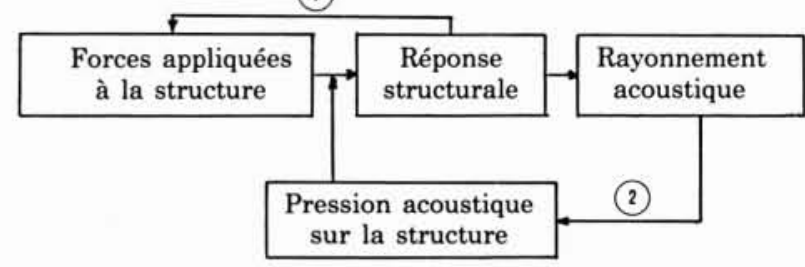

Le niveau (1) se rencontrerait, par exemple, dans le cas où, la structure présentant de grandes déformations, la couche limite qui est le phénomène excitateur, serait modifiée; on obtiendrait bien alors une modification du champ excitateur.

Ce type de couplage est négligé [10] dans le cas général où les déformations de la structure sont considérées comme faibles devant les épaisseurs de couche limite.

Le couplage (2) se produit lorsque le fluide baignant la structure est lourd (exemple de l'eau); il intervient alors, soit comme une inertie ajoutée augmentant la masse de la structure, soit comme un frottement additionnel dans le cas où de l'énergie acoustique est effectivement rayonnée.

Pour comprendre ce second effet, il y a lieu d'examiner quel est le champ de pression généré par une "frontière " présentant une déformation sinusoïdale décrite par:

$$
w(x, t)=W_{0} \cos (\omega t-k x)
$$

On peut montrer que tant que la longueur d'onde caractéristique de la déformée est inférieure à la longueur d'onde dans le milieu, le champ de pression généré est local et purement hydrodynamique; le fluide réagit en tant qu'inertie ajoutée.

Lorsque la longueur d'onde de la déformée devient supérieure à la longueur d'onde dans le milieu, une onde acoustique est générée; de l'énergie acoustique est émise et le fluide réagit en apportant de l'amortissement à la structure.

Cette réponse du fluide au mouvement de la structure peut être traduite comme une impédance fluide, $Z_{F}$, rapport entre la pression générée et la vitesse vibratoire de la frontière.

\subsection{Réponse vibratoire d'une structure à une excitation}

Faisons l'hypothèse que toute structure peut être considérée comme un assemblage de plaques planes de dimensions finies. Toute déformée d'une plaque élémentaire peut alors être décomposée sur la base des déformées modales de cette plaque qui, par exemple dans le cas de conditions aux limites du type appui simple, sont sinusoïdales.

Chaque déformée modale de rang $m n$ va alors générer un champ de pression assurant une nouvelle excitation de la structure comme on l'a vu ci-dessus, mais cette excitation n'a pas lieu d'être appropriée au mode $m n$ et elle va concerner la totalité des modes propres de la plaque; c'est ce que l'on appelle le couplage intermodal.
Moyennant certaines hypothèses [11] (plaques de grandes dimensions, modes élevés), le couplage intermodal peut être négligé, ce qui conduit à introduire une impédance fluide pour le mode $m n\left(Z_{F m n}\right)$ reliant le champ de pression et la vitesse vibratoire pour le mode $m n$.

La réponse vibratoire d'une plaque de dimensions finies à une excitation, se détermine de la façon suivante : l'excitation est tout d'abord composée sur la base modale, ce qui permet d'obtenir ce que l'on appelle la force généralisée, c'est-à-dire, pour un mode $m n$, la composante Fmn appropriée spatialement pour exciter le mode $m n$.

En l'absence de fluide lourd, la réponse de la plaque à l'excitation $F_{m n}$ s'obtient à partir de l'impédance mécanique $Z_{M m n}$ dépendant uniquement des caractéristiques géométriques et mécaniques de la plaque; lorsque la plaque est immergée, la réponse s'obtient ainsi :

$$
\mathscr{W}_{m n} \times Z_{M m n}=F_{m n}+F_{m n}^{\prime}
$$

où $F_{m n}^{\prime}$ est l'excitation supplémentaire due à la réaction du fluide :

$$
F_{m n}^{\prime}=Z_{F m n} \times \mathscr{W}_{m n}
$$

et $\mathscr{W}_{m n}$ est la réponse vibratoire.

Alors :

$$
\dot{W}_{m n}=\frac{F_{m n}}{Z_{M m n}+Z_{F m n}}
$$

L'explicitation complète des termes de cette formule très générale pourra être trouvée dans [12]. Néanmoins ce que l'on peut constater, c'est que l'amplitude des vibrations pour un mode donné sera d'autant plus importante que la fréquence d'excitation sera proche de la fréquence de résonance de la structure immergée (masse ajoutée) et que l'effort appliqué sera approprié à exciter le mode correspondant.

\subsection{Excitation par une couche limite turbulente}

Dans le schéma explicité ci-dessus, le modèle le mieux adapté à déterminer la réponse d'une structure à une excitation par couche limite turbulente est la représentation spatiotemporelle de Corcos telle qu'elle a été présentée ultérieurement.

Ce modèle permet d'obtenir [13] analytiquement la contribution de chaque nombre d'onde aux fluctuations de pression à la fréquence pulsation $\omega$, par l'intermédiaire de l'interspectre de pression $\phi_{p p}(k, \omega)$ dont une allure caractéristique est donnée sur la figure 8 .

Une telle forme montre qu'il existe une relation entre l'étendue spatiale de l'excitation et la fréquence: $k=$ $\omega / U_{\infty}$ où $U_{\infty}$ est la vitesse de l'écoulement. Ceci permet en particulier de vérifier s'il existe un risque d'excitation privilégiée d'un mode donné en comparant la dimension caractéristique de la déformée propre à l'étendue spatiale de l'excitation. 


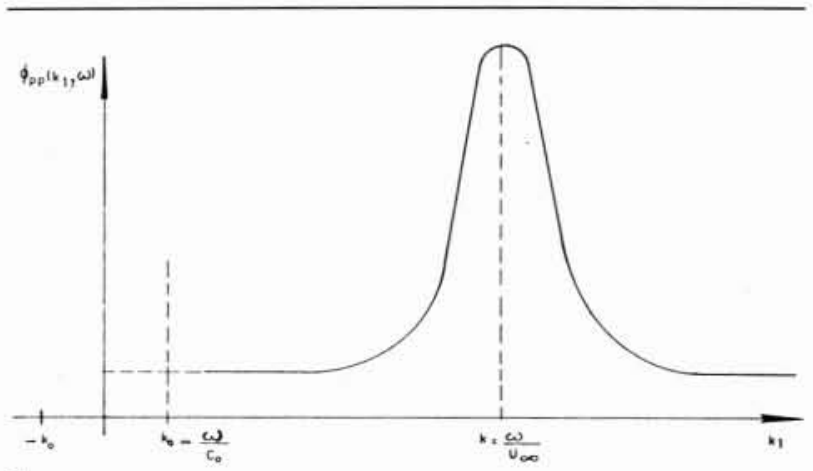

8. Spectre de pression en nombre d'ondes.

\section{Moyens de réduction du bruit de couche limite}

\subsection{Actions à la source}

Les équations (10) et (12) dérivées du modèle de Corcos et leurs correspondantes dans le domaine spatiotemporel mettent en évidence les paramètres importants qui gouvernent le phénomène sur lesquels il faut agir pour modifier le bruit de couche limite.

On peut agir sur la cohérence des sources :

Moins une source est cohérente, plus les valeurs des fonctions $\mathrm{A}$ et $\mathrm{B}$ du modèle de Corcos sont faibles et plus le niveau d'excitation efficace à une fréquence donnée va diminuer.

En ce sens, une turbulence homogène pleinement développée et déclenchée artificiellement apparaît plus souvent préférable à la recherche de conditions d'écoulements laminaires, très difficiles à obtenir la plupart du temps et aboutissant sur une zone de transition présentant un caractère d'organisation important.

Une paroi faiblement rugueuse peut donner de meilleurs résultats qu'une paroi lisse; mais cet effet peut être annihilé par l'augmentation du frottement moyen, qui contrôle le niveau global des fluctuations de pression.

La diminution des niveaux globaux est aussi une voie de réduction du niveau de bruit de proximité. Elle passe, suivant la formulation de Kraichnan par une réduction du frottement pariétal.

Celui-ci peut s'obtenir par plusieurs méthodes: injection pariétale de bulles, injection de polymère, parois souples, optimisation des profils de parois.

On se reportera à ce sujet aux nombreuses études spécialisées dans ce domaine.

\subsection{Action sur le transfert d'énergie hydrody- namique $\rightarrow$ Structure}

Le but recherché consiste à "provoquer" des perturbations dont les caractéristiques soient telles qu'elles se dissipent prioritairement à l'intérieur du fluide avant de communiquer leur énergie à la paroi. Celle-ci devra apparaître vis-à-vis des perturbations hydrodynamiques soit comme infiniment rigide, soit comme un milieu dissipatif.

Il est donc essentiel de savoir modéliser la réponse modale de la paroi, pour connaître les domaines en fréquences-nombre d'ondes où elle est la plus rigide (domaine autorisé) et ceux où elle est la plus souple (domaine interdit).

Une fois déterminé ce domaine, on jouera sur les paramètres hydrodynamiques $\left(U, \delta^{*}\right.$, taux de turbulence) de façon à éviter que les structures les plus énergétiques aient les caractéristiques dimensionnelles et fréquentielles comprises dans le domaine dangereux :

- on peut augmenter la dissipation turbulente des fluctuations à l'intérieur du fluide en plaçant par exem. ple des grilles ou des initiateurs de turbulences à des endroits judicieusement choisis;

- on peut aussi choisir la démarche inverse : on suppose connue la fonction d'excitation qui est le champ de pression turbulent convecté par l'écoulement. On concevra alors la paroi pour qu'elle ne réponde pas aux oscillations hydrodynamiques;

- on pourra couvrir la paroi d'un revêtement viscoélastique absorbant les fluctuations pour les dissiper en chaleur avant d'arriver au substrat solide;

- on pourra agir sur la structure elle-même (renfort, épaississement, liaisons mécaniques...) pour que ses modes propres soient situés en dehors de la zone dangereuse d'excitation hydrodynamique.

\section{Conclusions}

4.1. Les couches limites turbulentes sont une source de vibrations aléatoires à large bande (" bruit rose "). Il est possible au niveau de l'avant-projet de déterminer quelques paramètres caractéristiques de cette excitation :

si la vitesse d'écoulement est $U$

l'épaisseur de couche limite $\delta$

- la fréquence caractéristique du spectre en un point donné est de l'ordre de $U / \delta$

- le niveau global est de l'ordre de $3 C_{f} \rho U^{2} / 2$

où $C_{f}$ est le coefficient de frottement, $\rho$ la densité

- le nombre d'ondes caractéristique dans le sens de

l'écoulement est $k_{c} \# 1 / \delta$

- la célérité de propagation des perturbations est de l'ordre de $0,8 U$.

Avec des modèles plus sophistiqués, on peut aujourd'hui prévoir le champ de pression en paroi et la réponse de celle-ci, soit dans le domaine espace-temps (éléments ou différences finies), soit dans le domaine fréquencesnombre d'ondes.

4.2. Les conditions de transfert entre couche limite et paroi jouent un rôle essentiel.

La paroi se comporte comme un filtre mécanique complexe vis-à-vis de l'excitation hydrodynamique. Ce filtre est plus ou moins bien adapté et son amortissement plus ou moins important. Sa réponse, la vibration de structure, peut être schématisée par le produit.

$$
V=E . T
$$

où $E$ est l'excitation et $T$ la fonction de transfert; pour diminuer $V$, on agira sur $E$ ou $T$ ou les deux à la fois. Les résultats disponibles, tant expérimentaux que théoriques permettent de dégager les tendances et ordres de grandeur des actions à entreprendre en cas de besoin. 


\section{Discussion}

Président: M. R. DAT

\section{Références}

[1] Kraichnan. - Pressure fluctuations in turbulent flow over a plate, JASA, Vol. $28-\mathrm{n}^{\circ} 3$ (1956).

[2] KoVASNAY - Large scale motion intermittent region of a turbulent boundary layer, JMF, Vol. 41 - Part. 2.

[3] R. EMmerLiNG. - The instantaneous structure of the wall pressure under a turbulent boundary layer flow, Max Planck Institut für Strömungsforschung Bericht 9/1973 Göttingen.

[4] W.W. WILLMARTH - C.E. WOOLDRIDGE. - Measurements of the fluctuating pressure at the wall beneath a thick turbulent boundary layer, J.F.M., Vol. 14, pp.187-210 (1962).

[5] BuLL. - Wall pressure fluctuations in turbulent flows, $J M F$, Vol. 28 - part. 4 (1967).

[6] G.M. Corcos. - The structure of the turbulent pressure field in boundary layer flows, J.F.M., Vol. 18 - pp. 353-377 (1964).

[6] U. Schumann. - Numerical investigation of the wall pressure fluctuations in channel flow, Nuclear Engineering and Design. Vol. $32-\mathrm{n}^{\circ} 1$ (1975).

[8] F. FAHY. - Sound and structural vibration, Academic Press Inc - London - LTD (1985).

[9] W.K. BLAKE. - Mechanics of flow induced sound and vibration, Academic Press Inc (1986).

[10] G. RoBERT. - Modélisation et simulation du champ excitateur induit sur une structure par une couche limite turbu. lente. Thèse présentée devant l'Ecole Centrale de Lyon (1984).

[11] H.G. Davies. - Low-Frequency Random excitation of water loaded rectangular plates. J. Sound Vib., Vol. 15 (1) - pp. 107-126.

[12] M.C. JUNGER and D. FeIT. - Sound structures and their interactions, MIT Press, Cambridge, Massachussets and London, England (1972).

[13] E. Benarrous. - Contribution à l'étude des fluctuations de pression pariétale sous une couche limite turbulente, Thèse Université Claude Bernard - Lyon n 897 (1979).

\section{Adresses des auteurs}

Monsieur D. Leducq

Société Bertin

BP 3

78370 Plaisir

Tél. : 34.85.00.00

Monsieur R. Perret

Sté Alsthom - ACB - CERG

Voie privée Rhône-Poulenc

38800 Le Pont de Claix

Tél. : (76) 40.90 .40
M. SAGNER. - Dans l'exemple que vous avez décrit, il semble que ce sont les basses fréquences qui sont excitées. Quelle est votre interprétation de ce phénomène?

M. PERRET. - Ceci est dû :

- d'une part au fait que le spectre d'excitation présente une fréquence de coupure de l'ordre de grandeur du rapport de la vitesse d'écoulement sur l'épaisseur de la couche limite,

- d'autre part, à la gamme de fréquences observées sur la réponse qui correspond aux fréquences pour lesquelles la géométrie de l'excitation est voisine des allures des déformées modales de la structure mécanique.

M. SAGNER. - Que se passe-t-il pour les couches limites proches des décollements tourbillonnaires, notamment dans le cas des angles optimaux des diffuseurs?

M. LEDUCQ. - La couche limite dépend du gradient de pression et dans le cas que vous signalez la répartition spatiale des structures est très différente. L'approche que j'ai présentée correspond à une plaque plane; quand on arrive près du décollement les dimensions relatives des différentes couches seront fortement modifiées: la sous-couche visqueuse va presque disparaitre, la couche de paroi va prendre de l'importance et la couche de sillage peut devenir dans certains cas largement prépondérante.

M. SAGNER. - Les amplitudes des spectres seront-elles également modifiées?

M. LEDUCQ. - Les amplitudes seront différentes également car elles sont liées à l'organisation de la couche limite qui dépend du gradient de pression. La valeur du coefficient de la formule de Kraichnan peut varier entre 1.5 et 9, voire même plus

Par ailleurs, il est intéressant également de bien faire la distinction entre la plaque lisse et la plaque rugueuse. En ce qui me concerne, je considère que la plaque est rugueuse si les discontinuités de paroi sont de taille supérieure à la sous couche visqueuse, car des micro-sillages vont apparaître et les conditions du modèle que je viens de décrire ne sont plus valables; il faut alors utiliser de préférence un modèle d'obstacle.

M. THIRRIOT. - Comment expliquez-vous la renaissance du coefficient de corrélation à partir d'une certaine distance ? Ceci est-il dû à des vitesses différentes de propagation des perturbations dans la couche limite au voisinage de la paroi ?

M. LEDUCQ. - Effectivement, il existe deux familles de perturbations qui sont convectées à des vitesses différentes. L'apparition de la deuxième famille à une distance plus lointaine est due au fait que celle-ci est masquée au début par la première famille de perturbations.

M. THIRRIOT. - Vous avez signalé la possibilité de réduire le niveau de fluctuations de pression; pourriez-vous expliquer les raisons sur le plan hydrodynamique qui conduisent à cette diminution?

M. LEDUCQ. - D'après la formule de Kraichnan, l'amplitude des fluctuations de pression est directement liée au coefficient de frottement. Par conséquent il faut réduire le frottement. parmi les moyens de réduction du frottement à la paroi, l'injection de polymères agit en réorganisant l'écoulement au niveau de la sous couche visqueuse en le rendant anisotrope; on inhibe ainsi certains cisaillements. L'injection de micro-bulles est également envisageable bien que ce soit plus délicat; dans ce cas, on réorganise les tourbillons de Kline au niveau de la sous couche visqueuse en créant une relation diphasique. Dans le domaine des hautes fréquences, des parois souples peuvent être utilisées; celles-ci adaptent alors leurs déformations aux fluctuations de la sous couche elle-même, notamment en suivant la géométrie des tourbillons de Kline.

M. DAT. - Il faut un matériau très souple dans ce cas.

M. LEDUCQ. - En général, ce sont des mousses de polyuréthane qui sont utilisées. pour des cas extrêmes. 